\title{
Avaliação das concordâncias intra e interobservadores da nova classificação AO/OTA para fraturas da região trocantérica e do colo do fêmur*
}

\section{Evaluation of the Intraobserver and Interobserver Agreements of the New AO/OTA Classification for Fractures of the Trochanteric Region and the Femoral Neck}

\author{
Thiago Sampaio Busato $^{10}$ Daniel Baldasso $^{10}$ Gladyston Roberto Matioski Filho ${ }^{10}$ \\ Lucas Dias Godoi ${ }^{10}$ Marcelo Gavazzoni Morozowski ${ }^{1}{ }^{\circledR}$ Juan Rodolfo Vilela Capriotti ${ }^{10}$ \\ ${ }^{1}$ Grupo do Quadril, Centro de Reconstrução e Instituto de Pesquisa \\ Articular (CRIAR), Hospital Angelina Caron, Campina Grande do Sul, \\ Endereço para correspondência Thiago Sampaio Busato, MD, \\ Hospital Angelina Caron, Campina Grande do Sul, PR, Brasil \\ Paraná, Brasil \\ (e-mail: tsbusato@hotmail.com).
}

Rev Bras Ortop 2022;57(2):241-249.

\begin{abstract}
Resumo
Objetivo Neste estudo, investigamos a concordância intra e interobservador da nova classificação Arbeitsgemeinschaft für Osteosynthesefragen/Orthopaedic Trauma Association (AO/OTA) para fraturas da extremidade proximal do fêmur.

Métodos Foram selecionadas 100 radiografias do quadril de pacientes que sofreram fraturas da região trocantérica ou do colo do fêmur. Quatro ortopedistas cirurgiões de quadril e quatro residentes de ortopedia e traumatologia avaliaram e classificaram as fraturas segundo o novo sistema AO/OTA em duas ocasiões distintas. O coeficiente de kappa (k) foi utilizado para avaliar a concordância intra e interobservadores nos diferentes passos da classificação, a saber: tipo, grupo, subgrupo e qualificador.

Resultados Especialistas em cirurgia do quadril obtiveram concordância intraobservador quase perfeita de tipo, substancial para grupo e, apenas moderada para subgrupo e qualificadores. Os residentes tiveram desempenho inferior, com concordância substancial para o tipo, moderada para o grupo, e razoável para o subgrupo e qualificador. Na avaliação

Palavras-chave

- colo do fêmur

- fraturas do quadril

- classificação interobservadores dos especialistas, também se observou queda gradual da concordância entre tipo (quase perfeita) e grupo (moderada), que se mostrou ainda menor para subgrupo e qualificadores. Residentes tiveram uma concordância interobservadores substancial para tipo, moderada para grupo e razoável nas demais ramificações.
\end{abstract}

Trabalho desenvolvido no grupo de quadril Criar - Centro de Reconstrução e Instituto de Pesquisa Articular, Hospital Angelina Caron, Campina Grande do Sul, Paraná, Brasil. recebido

28 de Maio de 2020

aceito

01 de Dezembro de 2020

Publicado on-line

Dezembro 14, 2021
DOI https://doi.org/

10.1055/s-0041-1729939.

ISSN 0102-3616. (c) 2021. Sociedade Brasileira de Ortopedia e Traumatologia. All rights reserved.

This is an open access article published by Thieme under the terms of the Creative Commons Attribution-NonDerivative-NonCommercial-License, permitting copying and reproduction so long as the original work is given appropriate credit. Contents may not be used for commercial purposes, or adapted, remixed, transformed or built upon. (https://creativecommons.org/ licenses/by-nc-nd/4.0/)

Thieme Revinter Publicações Ltda., Rua do Matoso 170, Rio de Janeiro, RJ, CEP 20270-135, Brazil 


\begin{abstract}
Keywords

- femur neck

- hip fractures

- classification

Objective In the present study, we investigated the intra and interobserver agreement of the new Arbeitsgemeinschaft für Osteosynthesefragen/Orthopaedic Trauma Association (AO/OTA) classification for fractures of the proximal extremity of the femur.

Methods One hundred hip radiographs were selected from patients who suffered fractures of the trochanteric region or femoral neck. Four orthopedists, fellowship trained hip surgeons, and four orthopedic residents evaluated and classified fractures according to the new AO/OTA system on two separate occasions. The kappa (k) coefficient was used to evaluate intra and interobserver agreement in the different steps of the classification, namely: type, group, subgroup, and qualifier.

Results Hip surgery experts obtained almost perfect intraobserver agreement of type, substantial for group and, only moderate, for subgroup and qualifiers. The residents had lower performance, with substantial agreement for type, moderate for group, and reasonable for subgroup and qualifier. In the specialists' interobserver evaluation, there was also a gradual decrease in the agreement between type (almost perfect) and group (moderate), which was even lower for subgroup and qualifiers. Residents had a substantial interobserver agreement for type, moderate for group, and reasonable in the other branches.

Conclusion The new AO/OTA classification for fractures of the trochanteric region and femoral neck showed intra and interobserver agreements considered appropriate for type and group, with a drop in the subsequent branches, that is, for subgroup and qualifier. Still, in relation to the old AO/OTA classification, there was an improvement in the agreements for subgroup.
\end{abstract}

Conclusão A Nova Classificação AO/OTA para fraturas da região trocantérica e do colo do fêmur mostrou concordâncias intra e interobservadores consideradas adequadas para tipo e grupo com queda nas ramificações subsequentes ou seja para subgrupo e qualificador. Ainda assim em relação à classificação $\mathrm{AO} / \mathrm{OTA}$ antiga houve melhora nas concordâncias para subgrupo.

\section{Introdução}

As fraturas da extremidade proximal do fêmur causam grande morbidade e mortalidade a curto e médio prazo nos idosos, ${ }^{1-3}$ sendo que um terço dos pacientes evolui para óbito em um ano, e metade se torna dependente para locomoção. ${ }^{4,5}$ Estas fraturas também podem acometer pacientes jovens vitimados por trauma de alta energia. ${ }^{4} \mathrm{O}$ tratamento destas lesões requer a atuação de uma equipe multidisciplinar. ${ }^{6}$ Estas lesões têm tratamento eminentemente cirúrgico e, para que se defina o melhor tratamento, entre outros dados, é necessário que se classifique a fratura.

Um sistema ideal de classificação deve permitir a comunicação entre médicos, padronizar a terminologia para pesquisa e guiar a decisão do tratamento. ${ }^{6}$ Muitas tentativas de se criar um sistema de classificação de fraturas do fêmur proximal foram descritas, sendo as classificações de Garden, ${ }^{7}$ Evans, ${ }^{8}$ Boyd e Griffin, ${ }^{9}$ Tronzo ${ }^{10}$ e do Arbeitsgemeinschaft für Osteosynthesefragen/Orthopaedic Trauma Association (AO/OTA), ${ }^{11}$ as mais conhecidas. A classificação AO/OTA tem evidência favorável de confiabilidade na região trocantérica ${ }^{12-14}$ e no colo do fêmur ${ }^{15}$ quando comparada as outras classificações comumente utilizadas. Porém, suas ramificações adicionais tendem a diminuir a confiança interobservadores ${ }^{13,16}$ e requerem grande prática. ${ }^{16,17}$

Avalidação de uma classificação ocorre com a demonstração de alguns critérios: boa correlação clínica, adequação em termos de concordância e acurácia e também validação construtiva (relevância). ${ }^{18}$ Preocupações com a terminologia e linha de fluxo complexa da classificação AO/OTA prévia ${ }^{19}$ motivaram a modernização da classificação. Entretanto, não localizamos estudos na literatura sobre a confiabilidade desta nova versão.

Nesse sentido, este estudo teve como objetivos avaliar o grau de concordância intra e interobservadores em cada subdivisão sequencial da nova classificação AO/OTA para fraturas da extremidade proximal do fêmur, em observadores considerados experientes (cirurgiões de quadril) e observadores inexperientes (residentes de ortopedia).

\section{Materiais e Métodos}

Este estudo retrospectivo incluiu registros radiográficos de pacientes que sofreram fraturas da extremidade proximal do fêmur entre os anos de 2015 e 2019, tratados em um centro de referência em trauma ortopédico. Foram selecionados 100 
casos consecutivos para esta avaliação. 0 tamanho da amostra foi arbitrado com base em estudos prévios ${ }^{4-6,12,13,18}$ que utilizaram amostras menores para validação de classificações (entre 40-70 casos). O projeto foi previamente submetido e aprovado pela Comissão de Ética em Pesquisa da Instituição (CAAE: 30754120.7.0000.5226).

Os critérios de inclusão foram: presença de fratura da extremidade proximal do fêmur (osso 3, região anatômica 1 ), da região trocantérica (grupo A) ou do colo do fêmur (grupo B), em indivíduos esqueleticamente maduros. Fraturas da cabeça do fêmur (que são melhor avaliadas por tomografia axial computadorizada) e fraturas patológicas não foram incluídas neste estudo.

Cada participante recebeu as imagens digitalizadas de radiografia nas incidências em anteroposterior e perfil para análise. Não havia nenhuma informação do paciente ou do tratamento nas imagens utilizadas para classificação. Quatro ortopedistas especializados em cirurgia do quadril e 4 médicos residentes do $2^{\circ}$ ano de ortopedia e traumatologia classificaram todos os casos (de maneira sequencial e ininterrupta, sem limitação de tempo) em 2 momentos distintos, com intervalo de 4 semanas. No início das avaliações, a descrição detalhada da nova classificação e suas imagens ilustrativas foram disponibilizadas aos avaliadores para aprendizagem do sistema. Cada avaliação foi realizada individualmente e não foi permitida a guarda das respostas ou a discussão dos resultados entre os mesmos.
A confiabilidade interobservador foi determinada através da primeira resposta entre os avaliadores e a intra observador através de nova avaliação quatro semanas após a inicial. Este intervalo foi utilizado para se reduzir o risco de viés de memória. Os dados foram coletados e armazenados em planilhas para análise estatística. Para avaliar a concordância intraobservador foi calculado o coeficiente kappa de Cohen e para avaliar a concordância interobservadores foi calculado o coeficiente kappa de Fleiss. Para as análises foram usados o software SPSS Statistics for Windows, Version 20.0 (IBM Corp., Armonk, NY, EUA) e a Online kappa Calculator www.statisticssolutions.com. A avaliação de concordância incluiu 4 etapas: tipo de fratura (A- trocantérica ou B- colo), grupo (1, 2 ou 3), subgrupos (1, 2 ou 3-exceto no tipo $\mathrm{B} 3$, que não possui subgrupos) e também seus qualificadores quando disponíveis na classificação, ou seja, apenas nas A.1.1 (N ou O) ou B2 1, 2 ou 3 (P, Q ou R). A - Tabela 1 e a - Tabela 2 ilustram as diferenças entre as classificações AO/OTA antiga e a nova.

Embora o grau de concordância tenha formas distintas de interpretação, ${ }^{19}$ adotamos a proposta clássica de Landis e Koch, ${ }^{20}$ com valores entre 0.00 e 0.20 considerados como concordância leve; 0.21 e 0.40 concordância razoável; 0.41 e 0.60 concordância moderada; 0.61 e 0.80 concordância substancial, e 0.81 e 1.00 concordância quase perfeita (ou excelente).

Na amostra populacional estudada, a média de idade foi de 77,71 anos (variando de 57 a 98 anos, desvio padrão de

Tabela 1 Comparação ilustrada entre os sistemas AO/OTA (Grupo A)

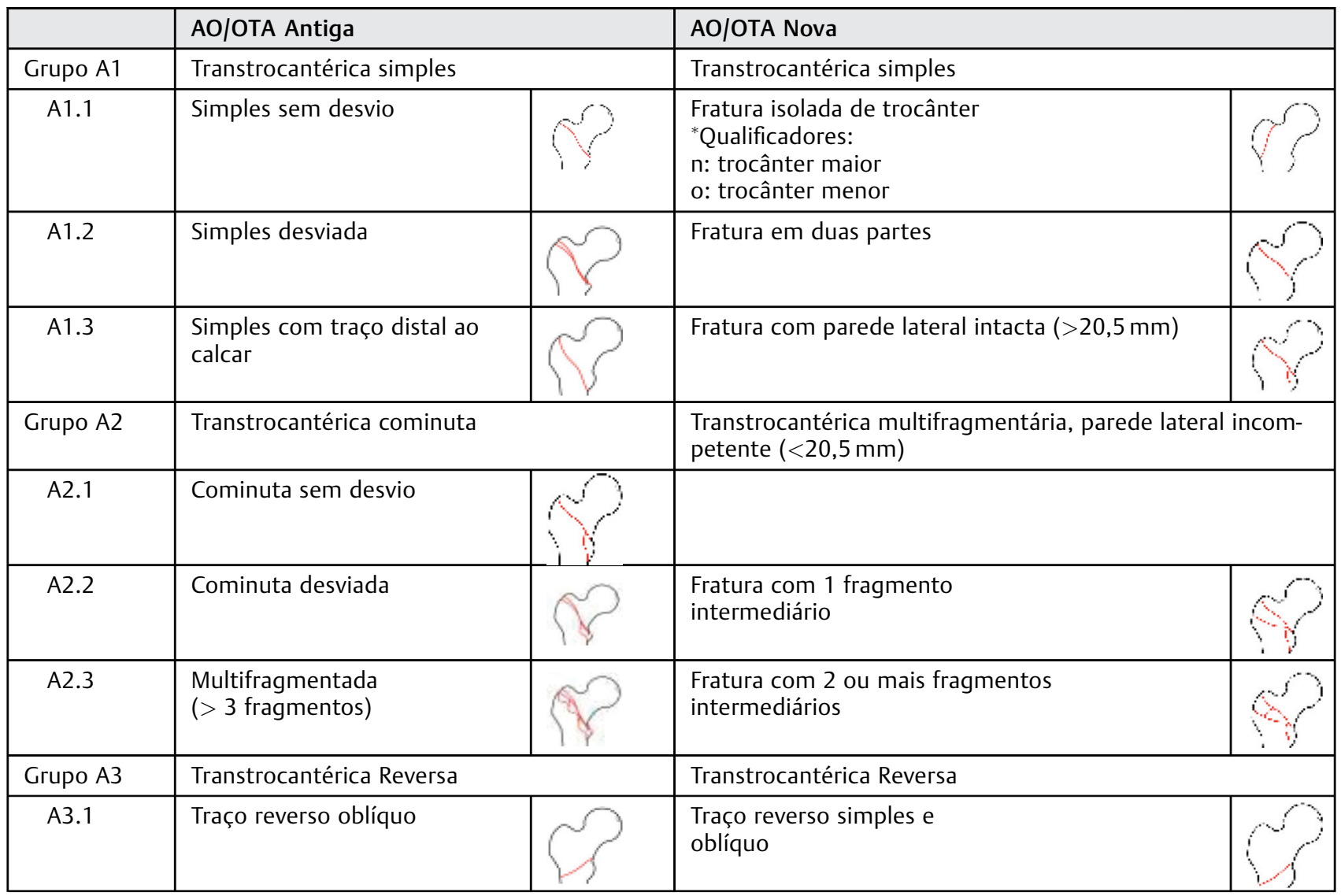


Tabela 1 (Continued)

\begin{tabular}{|l|l|c|l|}
\hline & \multicolumn{2}{|l|}{ AO/OTA Antiga } & \multicolumn{2}{l|}{ AO/OTA Nova } \\
\hline A3.2 & Traço reverso transverso & Traço reverso simples e transverso \\
\hline A3.3 & $\begin{array}{l}\text { Traço reverso com fratura } \\
\text { do pequeno trocanter }\end{array}$ & $\begin{array}{l}\text { Traço reverso com cunha ou } \\
\text { multifragmentária }\end{array}$ \\
\hline
\end{tabular}

Tabela 2 Comparação ilustrada entre os sistemas AO/OTA (Grupo B)

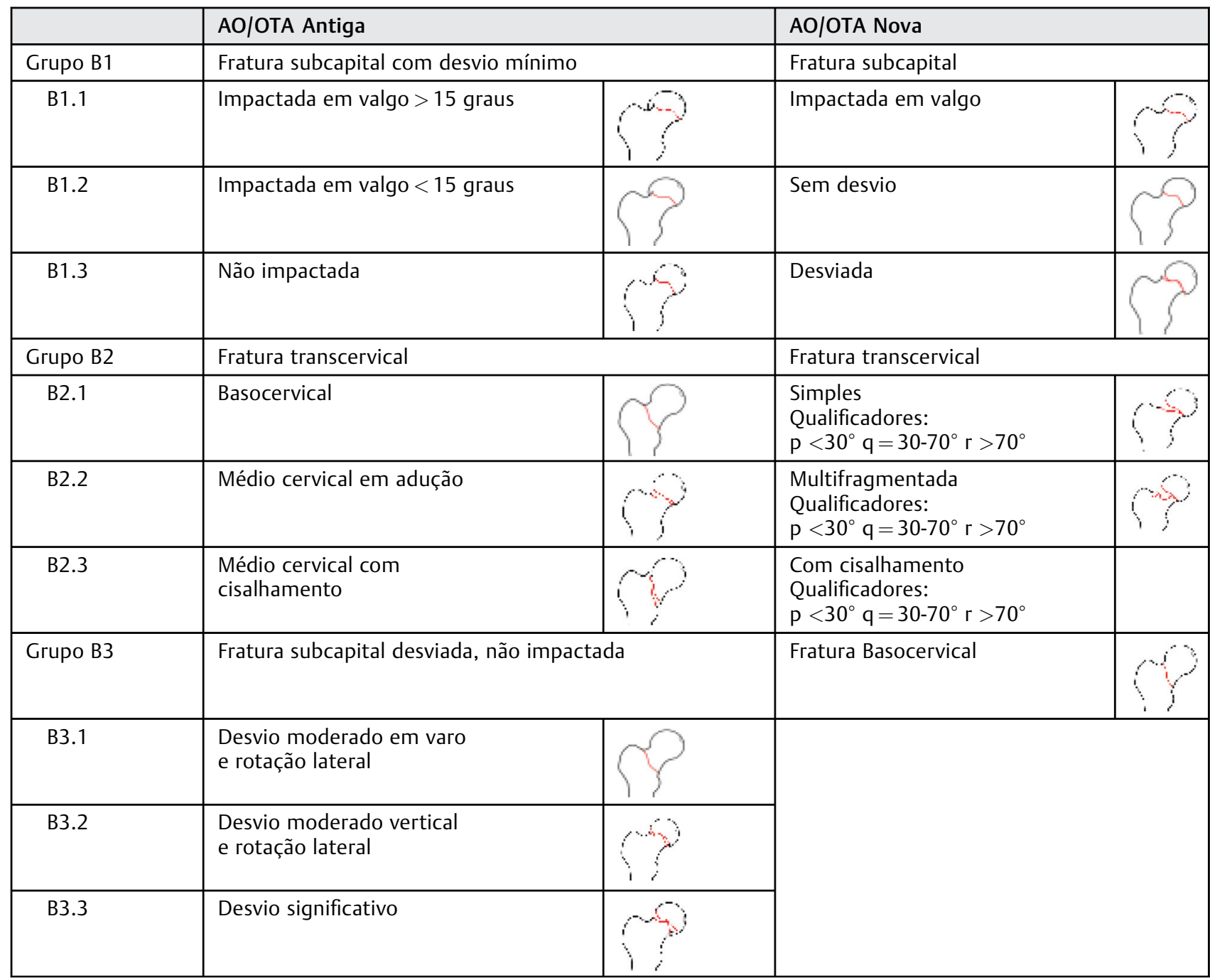

10,12). O sexo feminino foi predominante, com $63 \%$ dos casos, e o lado direito teve um caso a mais de fratura (51\%).

\section{Resultados}

\section{Concordância intraobservador}

Na avaliação repetida com intervalo de 4 semanas, a concordância intraobservador de tipo foi quase perfeita para os especialistas com $k$ médio de 0.92 , enquanto que a dos residentes foi substancial ( $k$ médio 0.77 ). Já na classificação de grupo, os especialistas apresentam uma concordância substancial ( $k$ médio 0.68 ) e os residentes moderada ( $k$ médio 0.44). Para subgrupo, a concordância dos especialistas foi moderada ( $k$ médio 0.52 ), e a dos residentes foi razoável ( $k$ médio 0.28). Por fim, em relação aos qualificadores, a concordância dos especialistas também foi moderada ( $k$ médio 0.50 ) e a dos residentes foi razoável ( $k$ médio $0.27)$. 
De modo geral, os especialistas apresentam desempenho melhor que os residentes. Observa-se ainda ( - Figura 1) que os coeficientes vão decrescendo à medida que se seguem as ramificações da classificação. A - Tabela 3 descreve detalhadamente os achados intraobservadores.

\section{Concordância interobservador}

Considerando a primeira rodada como padrão (usado na maioria dos estudos) para a avaliação interobservador, temos uma concordância no tipo de $93,67 \%$ para os especialistas ( $k 0.87$, quase perfeita) e $90,17 \%$ para os residentes ( $k$ 0.80 , substancial). No grupo, a concordância foi de $60,83 \%$ para os especialistas ( $k 0.53$, moderada) $55,5 \%$ para os residentes ( $k 0.47$, moderada). Avançando para subgrupo a concordância caiu para $44,5 \%$ entre os especialistas ( $k 0.41$, moderada) e $42,7 \%$ para os residentes ( $k 0.39$, razoável). Finalmente, nos qualificadores, a concordância foi $42,67 \%$ para os especialistas ( $k$ 0.40, razoável) e $41,0 \%$ para os residentes ( $k 0.39$, razoável).

A - Tabela 4 detalha os resultados interobservadores. Tanto os especialistas quanto os residentes tiveram decréscimo dos coeficientes à medida que a classificação se ramifica. No entanto, na transição subgrupo-qualificador, o decréscimo da concordância foi pouco significativo. Na primeira rodada, os residentes alcançaram coeficientes sempre abaixo dos coeficientes dos especialistas (- Figura 2), mas na segunda rodada, os residentes apresentam uma concordância maior entre si do que os especialistas (- Figura $\mathbf{3}$ ).
Tabela 3 Coeficiente kappa de concordância intraobservador

\begin{tabular}{|l|l|l|l|l|}
\hline \multicolumn{5}{|l|}{ kappa (Cohen) } \\
\hline Especialista & Tipo & Grupo & Subgrupo & Qualificador \\
\hline 1 & 0,972 & 0,705 & 0,607 & 0,607 \\
\hline 2 & 0,972 & 0,589 & 0,376 & 0,338 \\
\hline 3 & 0,894 & 0,747 & 0,599 & 0,600 \\
\hline 4 & 0,851 & 0,713 & 0,500 & 0,459 \\
\hline Residente & & & & \\
\hline 1 & 0,828 & 0,468 & 0,298 & 0,300 \\
\hline 2 & 0,851 & 0,443 & 0,259 & 0,260 \\
\hline 3 & 0,806 & 0,640 & 0,421 & 0,391 \\
\hline 4 & 0,608 & 0,230 & 0,153 & 0,144 \\
\hline
\end{tabular}

\section{Discussão}

Na classificação AO/OTA prévia, alguns padrões de fratura ocorriam tão raramente que não havia necessidade de uma codificação exclusiva para estes. A terminologia era foco de confusão, devido a grande variedade de termos para fraturas similares. Havia, ainda, dificuldade em definir-se as fraturas do grupo A2. Na nova classificação, as definições e os códigos foram atualizados e simplificados. As fraturas do colo foram reorganizadas e o qualificador de Pauwels agregado para

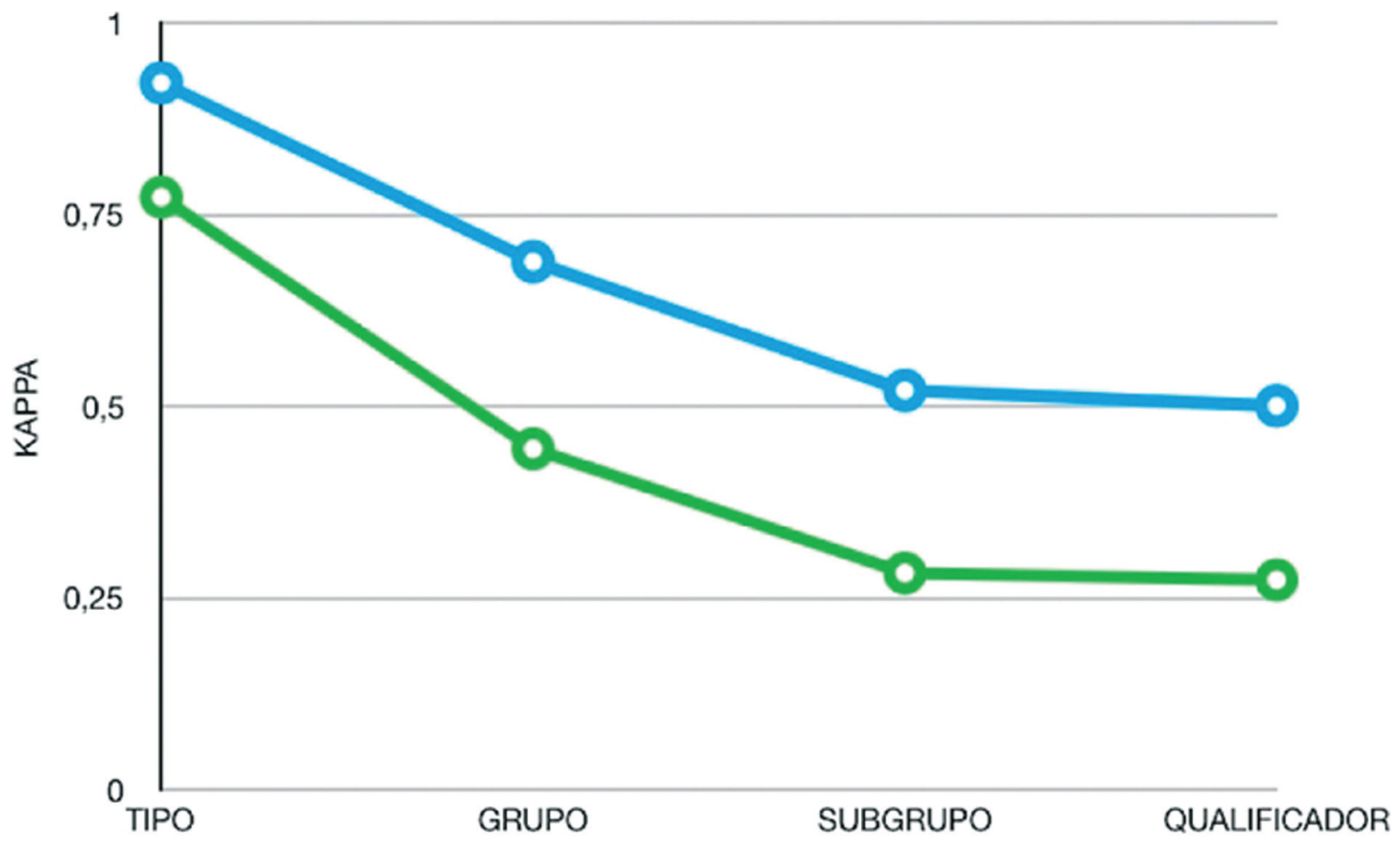

Fig. 1 Comparativo entre especialistas e residentes do coeficiente médio kappa (intraobservador). 
Avaliação da concordância intra e interobservadores da nova classificação AO/OTA Busato et al.

Tabela 4 Coeficiente kappa de concordância (Fleiss) interobservador (especialistas e residentes)

\begin{tabular}{|c|c|c|c|c|}
\hline & \multicolumn{2}{|l|}{ Avaliação 1} & \multicolumn{2}{|l|}{ Avaliação 2} \\
\hline TIPO & \% concordância & Kappa & \% concordância & Kappa \\
\hline Especialistas & 93,67 & 0,87 & 97,00 & 0,94 \\
\hline Residentes & 90,17 & 0,80 & 94,50 & 0,89 \\
\hline \multicolumn{5}{|l|}{ GRUPO } \\
\hline Especialistas & 60,83 & 0,53 & 58,83 & 0,51 \\
\hline Residentes & 55,50 & 0,47 & 69,50 & 0,63 \\
\hline \multicolumn{5}{|l|}{ SUBGRUPO } \\
\hline Especialistas & 44,50 & 0,41 & 39,67 & 0,35 \\
\hline Residentes & 42,67 & 0,39 & 57,67 & 0,55 \\
\hline \multicolumn{5}{|c|}{ QUALIFICADOR } \\
\hline Especialistas & 42,67 & 0,40 & 37,33 & 0,35 \\
\hline Residentes & 41,00 & 0,39 & 57,17 & 0,55 \\
\hline
\end{tabular}

melhor definição de instabilidade, especialmente em fraturas de alta energia. ${ }^{19}$

Um sistema de classificação de fraturas deve ter concordância adequada entre o mesmo observador em oportunidades diferentes (intraobservador) e entre diferentes observadores na mesma oportunidade (interobservador). ${ }^{6,17,18}$ o coeficiente kappa ( $k$ ) é um dos métodos mais usados para avaliar a acurácia diagnóstica de um sistema de classificação; tendo seu cálculo ajustado para possíveis coincidências ao acaso. ${ }^{3}$

Neste estudo, para os especialistas, a concordância intraobservador foi quase perfeita para tipo, substancial para grupo e moderada para subgrupo e qualificador, enquanto os residentes tiveram um desempenho abaixo em todas as divisões.

Comparar as concordâncias interobservadores nas duas rodadas foi um aspecto interessante desta pesquisa. Uma

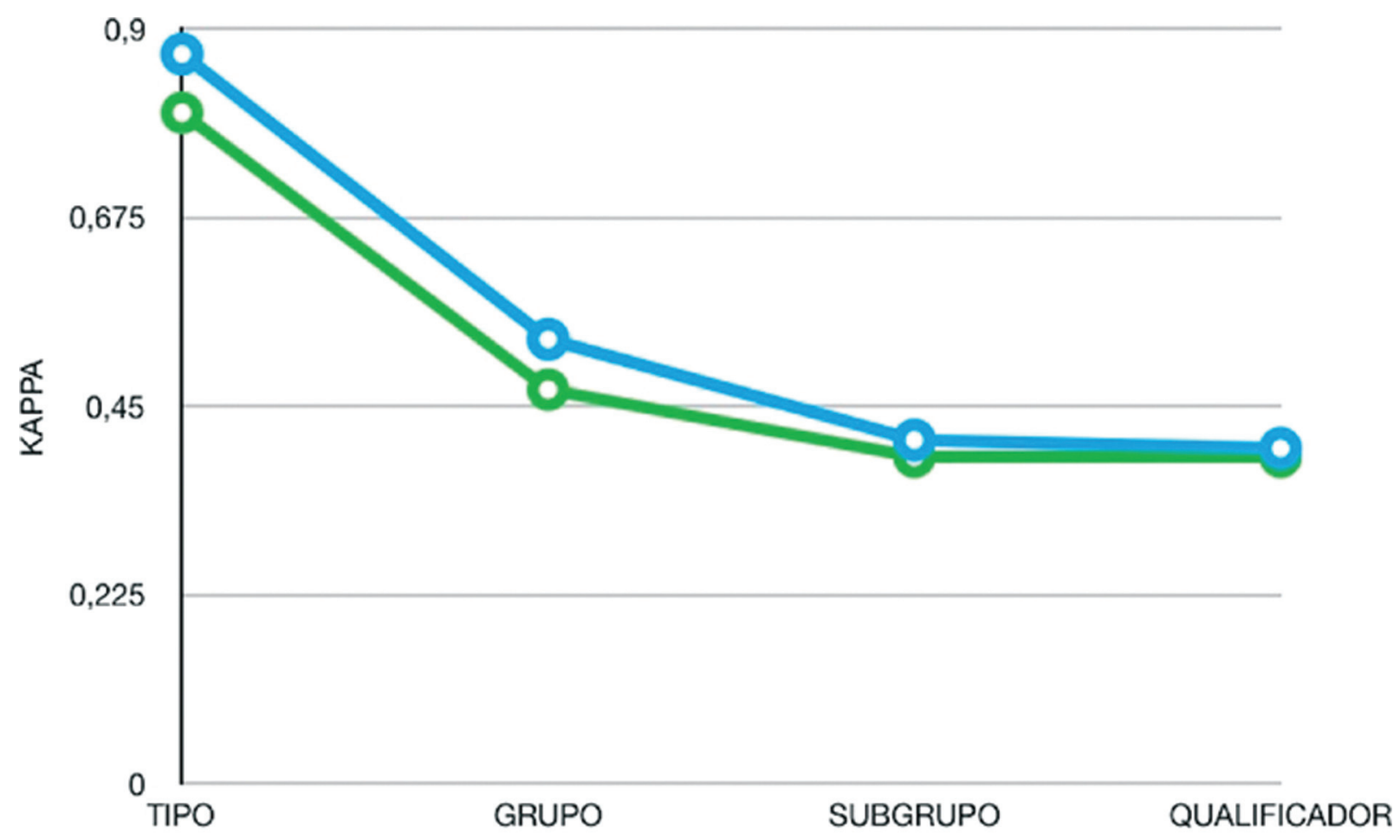

\section{ESPECIALISTAS}

\section{RESIDENTES}

Fig. 2 Concordância interobservadores na primeira avaliação. 


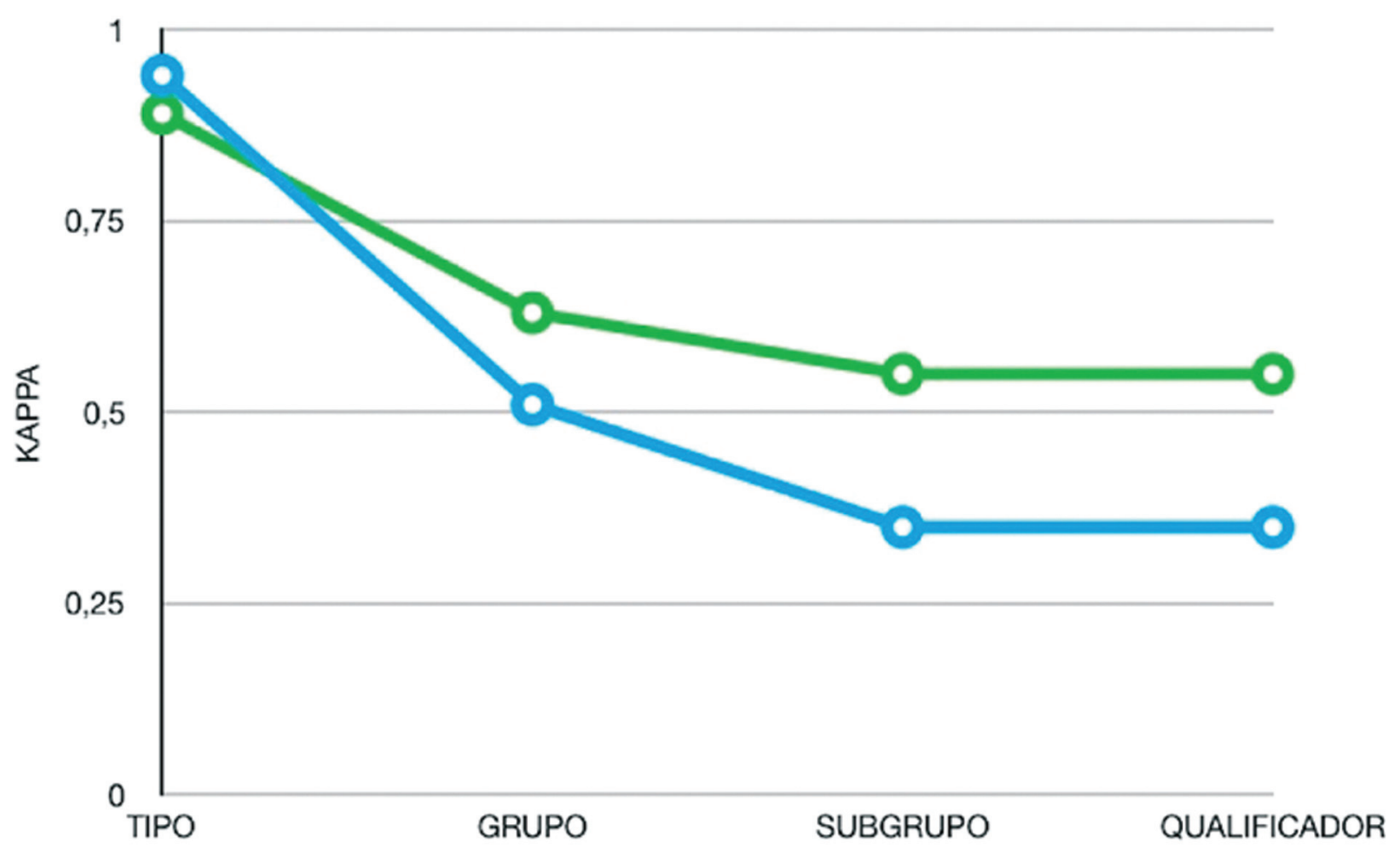

\section{ESPECIALISTAS}

\section{RESIDENTES}

Fig. 3 Concordância interobservadores na segunda avaliação.

observação curiosa é que a concordância interobservadores dos residentes aumentou entre as rodadas, o que talvez tenha indicado a capacidade de aprendizado da nova classificação.

Estudos sobre o sistema AO/OTA anterior obtiveram resultados similares aos obtidos em nosso estudo, mas com pequenas variações. $\mathrm{O}$ estudo de Pervez et al. ${ }^{3}$ obteve um $k$ médio entre seus observadores de 0.62 para grupo, maior do que o observado em nosso estudo ( $k 0,53$ concordância moderada) e de 0.33 nos subgrupos, menor do que encontramos neste estudo ( $k 0,41$ - concordância moderada). Urrutia et al. ${ }^{6}$ obteve concordância moderada, como nossos resultados, entre seus 9 avaliadores para os grupos, e apenas razoável para os subgrupos menor do que em nosso estudo ( $k$ 0,41 - concordância moderada). Mattos et al. ${ }^{4}$ também obteve resultados semelhantes com AO/OTA e Tronzo. Já Schwartsmann et al., ${ }^{14}$ em um estudo também englobando ortopedistas e residentes, obteve concordância moderada (0.60) para grupo, similar à presente pesquisa, e razoável (0.34) para os subgrupos, menor do que a observada no presente estudo $(\mathrm{k} 0,41)$. Outro estudo interessante, ${ }^{15}$ com 100 fraturas do colo do fêmur, graduou a classificação de Garden ${ }^{7}$ como de concordância interobservadores apenas razoável, que aumentou para moderada ao se simplificar o critério para fraturas desviadas ou não. Isto indica que, em determinadas fraturas, até mesmo uma classificação bastante simples pode gerar concordância apenas moderada.

Em resumo, em relação à classificação antiga, no presente estudo obtivemos concordância similar a da literatura para tipo e grupo e melhor concordância para subgrupo, enquanto qualificadores não eram disponíveis na classificação antiga. Isso indica que o novo sistema obteve sucesso ao trazer maior concordância nos subgrupos, que foram mais extensamente modificados.

Avaliando a questão de experiência dos examinadores na classificação AO/OTA, Crijins et al. ${ }^{16}$ não observaram diferença entre 65 cirurgiões divididos entre mais e menos experientes de acordo com as variáveis de tempo de prática ( $>$ ou $<17$ anos), tempo de trabalho dedicado ao trauma ( $>$ ou $<80 \%$ ) e fraturas tratadas por ano $(>$ ou $<50$ ). Em uma analogia com nosso estudo, avaliamos que os residentes se equipararam aos especialistas na segunda avaliação, indicando uma curva rápida de aprendizado neste sistema. Fung et al. ${ }^{21}$ também notou que residentes mais experientes, na sua parte final de treinamento, tinham uma avaliação melhor que os menos experientes, indicando o aprendizado da classificação antiga.

Sobre o novo sistema AO/OTA especificamente, é interessante notar a eliminação do subgrupo A2.1, o que pode auxiliar na distinção de padrões estáveis (grupo A1), dos instáveis (grupos A2 e A3). Estudos do sistema prévio ${ }^{11}$ que tentaram discernir até que ponto uma fratura trocantérica era estável tiveram resultados algo conflitantes. Radaideh et al. ${ }^{22}$ em um estudo sobre uso de hastes céfalo-medulares, definia os grupos A2 e A3 como instáveis, assim com Zhang et al. ${ }^{23}$ Já Knobe et al. ${ }^{24}$ citam que os grupos A2 e A3 são geralmente considerados instáveis na literatura, porém em 
uma avaliação direta a fratura do trocânter menor era o principal critério de instabilidade para $82 \%$ dos cirurgiões, entre outros fatores considerados (fratura do trocânter maior, fratura da parede lateral e traço reverso). Outro estudo $^{25}$ considerava com instáveis os subgrupos A2.1, A2.2 e A3.3.

A classificação atual tem a integridade da parede lateral (largura superior a $20,5 \mathrm{~mm}$ ) como o padrão de divisão entre o grupo A1 e A2. O racional para esta divisão foi inicialmente descrito por Gottfried ${ }^{26}$ e Palm et al., ${ }^{27}$ que definiram a parede lateral como importante estrutura para suporte de implantes. Posteriormente, Hsu et al. ${ }^{28}$ conseguiram avaliar qual a espessura da parede lateral seria necessária para síntese com placa-parafuso deslizante com segurança, o que motivou a mudança atual da classificação. Outros estu$\operatorname{dos}^{29}$ revisaram o assunto e trouxeram estratégias para reconstrução da parede lateral mesmo com o uso de síntese intramedular. Com base nesta revisão da literatura e as dificuldades de classificação em subgrupos, achamos interessante a divisão dos grupos a partir de A2 (inclusive) como parâmetro para considerar uma fratura instável que requer acurada técnica de redução e síntese intramedular.

Nosso estudo evidencia as dificuldades em sistemas de classificação para fraturas da extremidade proximal do fêmur. Apesar destas dificuldades, este sistema demonstrou vantagens em relação ao seu antecessor ${ }^{11}$ ao simplificar uma divisão para fraturas trocantéricas instáveis ao nível de grupo (A2 e A3) podendo facilitar uma eventual escolha de implante e técnicas de redução. Nas fraturas do colo (tipo B), a nova subdivisão é mais simples do que os complexos subgrupos prévios (-Tabela 1), e também engloba o qualificador de Pauwels. Adicionalmente, verificamos através da literatura, uma confiabilidade superior da nova classificação AO/OTA quando comparada com outros sistemas muito difundidos (Garden, Evans, Boyd, Tronzo).

No presente estudo, buscamos uma metodologia consistente para avaliação de uma classificação de fraturas, tendo como pontos fortes o tamanho e representatividade da amostra (maior do que estudos prévios, sendo que todos os padrões da nova classificação foram identificados por pelo menos um avaliador), além de um número adequado de observadores para melhor confiabilidade do coeficiente de Kappa. ${ }^{20}$ A metodologia de trabalhos de confiabilidade de classificações ortopédicas foi examinada por Audigé et al., ${ }^{18} \mathrm{e}$ o presente estudo engloba todos os critérios de qualidade descritos. Além disso, não foi encontrado nenhum estudo com avaliação deste novo sistema nas bases de dados do Pubmed, Medline e Scielo, o que traz dados inéditos e relevantes sobre esta classificação muito popular entre cirurgiões ortopédicos. Outro ponto interessante foi a evolução da concordância interobservadores notada entre médicos residentes entre as avaliações, indicando o aprendizado do sistema. Um ponto deficitário nesta pesquisa foi a inclusão de especialistas de quadril em comparação com apenas quatro residentes do segundo ano. Talvez, a inclusão também de residentes do primeiro e do terceiro ano pudesse demonstrar ainda melhor o processo de aprendizado.
Como outra possível limitação do presente estudo, destacamos a idade média relativamente alta da amostra, indicando uma característica típica do perfil da população do hospital onde este foi realizado. Porém, embora exista risco potencial de não se representarem algumas fraturas mais específicas do trauma de alta energia, mais comum nos mais jovens, ainda assim, todos os padrões da nova classificação AO/OTA foram identificados em algum momento durante o estudo. Se buscássemos outros pacientes mais jovens para incluir na amostra, incorreríamos em viés de seleção. Além disso, estudos desta natureza possuem limitações inerentes ao seu desenho, como potencial vício de memória, que consideramos baixo devido ao grande número de casos, a complexidade da classificação e pelo tempo transcorrido entre as avaliações.

O novo sistema AO/OTA tem uma confiabilidade moderada interobservadores e substancial intraobservadores para avaliadores experientes. Médicos residentes são capazes de atingir os mesmos níveis de concordância após curto período de aprendizado. Mais estudos são necessários para avaliar sua capacidade em relação à indicação de tratamento (especialmente sobre tipo de síntese) e de prognóstico.

\section{Conclusão}

A nova classificação AO/OTA para fraturas da região trocantérica e do colo do fêmur mostrou concordâncias intra- e interobservadores adequadas para tipo e grupo, com queda nas ramificações subsequentes, ou seja, subgrupo e qualificador. Ainda assim, em relação à classificação AO/OTA antiga, houve melhora nas concordâncias para subgrupos.

\section{Suporte Financeiro}

Não houve suporte financeiro de fontes públicas, comerciais, ou sem fins lucrativos.

\section{Conflito de Interessses}

Os autores declaram não haver conflito de interesses.

\section{Referências}

1 Rapp K, Becker C, Lamb SE, Icks A, Klenk J. Hip fractures in institutionalized elderly people: incidence rates and excess mortality. J Bone Miner Res 2008;23(11):1825-1831

2 Martinez-Reig M, Ahmad L, Duque G. The orthogeriatrics model of care: systematic review of predictors of institutionalization and mortality in post-hip fracture patients and evidence for interventions. J Am Med Dir Assoc 2012;13(09):770-777

3 Pervez H, Parker MJ, Pryor GA, Lutchman L, Chirodian N. Classification of trochanteric fracture of the proximal femur: a study of the reliability of current systems. Injury 2002;33(08):713-715

4 Mattos CA, Jesus AAK, Floter MS, Nunes LFB, Sanches BB, Zabeu JLA. Reprodutibilidade das classificações de Tronzo e AO para fraturas transtrocanteriana. Rev Bras Ortop 2015;50(05): 495-500

5 Oliveira FAS, Basile R, Pereira BCB, Cunha RLL. Avaliação da reprodutibilidade da classificação de Tronzo para fraturas intertrocantéricas do fêmur. Rev Bras Ortop 2014;49(06):581-585

6 Urrutia J, Zamora T, Besa P, Zamora M, Schweitzer D, Klaber I. Inter and intra-observer agreement evaluation of the $\mathrm{AO}$ and the 
Tronzo classification systems of fractures of the trochanteric area. Injury 2015;46(06):1054-1058

7 Garden RS. Low-angle fixation in fractures of the femoral neck. J Bone Joint Surg Br 1961;43(03):647-663

8 Evans EM. The treatment of trochanteric fractures of the femur. J Bone Joint Surg Br 1949;31B(02):190-203

9 Boyd HB, Griffin LL. Classification and treatment of trochanteric fractures. Arch Surg 1949;58(06):853-866

10 Tronzo RG. Surgery of the Hip Joint. Philadelphia: Lea \& Febiger; 1973

11 Müller ME, Nazarian S, Koch P. Classification AO des fractures. Tome I. Les os longs. Berlin: Springer-Verlag; 1987

12 Jin WJ, Dai LY, Cui YM, Zhou Q Jiang LS, Lu H. Reliability of classification systems for intertrochanteric fractures of the proximal femur in experienced orthopaedic surgeons. Injury 2005; 36(07):858-861

13 Schipper IB, Steyerberg EW, Castelein RM, van Vugt AB. Reliability of the AO/ASIF classification for pertrochanteric femoral fractures. Acta Orthop Scand 2001;72(01):36-41

14 Schwartsmann CR, Boschin LC, Moschen GM, Gonçalves R, Ramos AS, Gusmão PD. Classificação das fraturas trocantéricas: avaliação da reprodutibilidade da classificação AO. Rev Bras Ortop 2006;41 (07):264-267

15 Van Embden D, Rhemrev SJ, Genelin F, Meylaerts SA, Roukema GR. The reliability of a simplified Garden classification for intracapsular hip fractures. Orthop Traumatol Surg Res 2012;98(04):405-408

16 Crijns TJ, Janssen SJ, Davis JT, Ring D, Sanchez HBScience of Variation Group. Reliability of the classification of proximal femur fractures: Does clinical experience matter? Injury 2018;49(04): 819-823

17 Gusmão PD, Mothes FC, Rubin LA, Gonçalves RZ, Teloken MA, Schwartsmann CR. Avaliação da reprodutibilidade da classificação de Garden para fraturas do colo femoral. Rev Bras Ortop 2002; 37(09):381-386

18 Audigé L, Bhandari M, Kellam J. How reliable are reliability studies of fracture classifications? A systematic review of their methodologies. Acta Orthop Scand 2004;75(02):184-194
19 Hartline BE, Achor TS. Use of the 95-degree Angled Blade Plate to Treat a Proximal Femur Fracture. J Orthop Trauma 2018;32 (Suppl 1):S26-S27

20 Landis JR, Koch GG. The measurement of observer agreement for categorical data. Biometrics 1977;33(01):159-174

21 Fung W, Jonsson A, Buhren V, Bhandari M. Classifying intertrochanteric fractures of the proximal femur: does experience matter? Med Princ Pract 2007;16(03):198-202

22 Radaideh AM, Qudah HA, Audat ZA, Jahmani RA, Yousef IR, Saleh AAA. Functional and Radiological Results of Proximal Femoral Nail Antirotation (PFNA) Osteosynthesis in the Treatment of Unstable Pertrochanteric Fractures. J Clin Med 2018;7(04):78

23 Zhang WQ, Sun J, Liu CY, Zhao HY, Sun YF. Comparing the Intramedullary Nail and Extramedullary Fixation in Treatment of Unstable Intertrochanteric Fractures. Sci Rep 2018;8(01):2321

24 Knobe M, Gradl G, Ladenburger A, Tarkin IS, Pape HC. Unstable intertrochanteric femur fractures: is there a consensus on definition and treatment in Germany? Clin Orthop Relat Res 2013;471 (09):2831-2840

25 Cech O, Kostál R, Váchal J. [Unstable pertrochanteric fractures, biomechanic, classification and therapy.]. Acta Chir Orthop Traumatol Cech 2000;67(01):17-27

26 Gotfried Y. The lateral trochanteric wall: a key element in the reconstruction of unstable pertrochanteric hip fractures. Clin Orthop Relat Res 2004;(425):82-86

27 Palm H, Jacobsen S, Sonne-Holm S, Gebuhr PHip Fracture Study Group. Integrity of the lateral femoral wall in intertrochanteric hip fractures: an important predictor of a reoperation. J Bone Joint Surg Am 2007;89(03):470-475

28 Hsu CE, Shih CM, Wang CC, Huang KC. Lateral femoral wall thickness. A reliable predictor of post-operative lateral wall fracture in intertrochanteric fractures. Bone Joint J 2013;95-B (08):1134-1138

29 Tawari AA, Kempegowda H, Suk M, Horwitz DS. What makes an intertrochanteric fracture unstable in 2015? Does the lateral wall play a role in the decision matrix?. J Orthop Trauma 2015;29 (Suppl 4):S4-S9 